\title{
Evaluación de la competencia transversal CT04 "Innovación, creatividad y emprendimiento" en el Grado en Administración y Dirección de Empresas de la Universitat Politècnica de València
}

\author{
Baviera-Puig, Amparoa; García García, Fernando ${ }^{\text {b }}$; Hervás Oliver, Jose Luis ${ }^{c}$; \\ Loras, Joaquín ${ }^{\mathrm{d}}$; de-Miguel-Molina, Blanca ${ }^{\mathrm{e}}$ y Palau-Ramírez, Felipe ${ }^{\mathrm{f}}$ \\ aDepartamento de Economía y Ciencias Sociales, Universitat Politècnica de València, España, ambapui@upv.es, \\ bDepartamento de Economía y Ciencias Sociales, Universitat Politècnica de València, España, fergarga@esp.upv.es, \\ 'Departamento de Organización de Empresas, Universitat Politècnica de València, España, jose.hervas@omp.upv.es, \\ ${ }^{\mathrm{d}}$ Departamento de Organización de Empresas, Universitat Politècnica de València, España, jloras@doe.upv.es, \\ eDepartamento de Organización de Empresas, Universitat Politècnica de València, España, bdemigu@omp.upv.es y \\ fDepartamento de Economía y Ciencias Sociales, Universitat Politècnica de València, España, fpalau@upv.es.
}

\begin{abstract}
The UPV has defined a skill profile for all students graduated from the UPV in order to improve their professional performance. This profile consists of 13 generic skills. The objective of this work is to present different assessment methodologies of the GS-04. "Innovation, creativity and entrepreneurship", carried out throughout the Degree in Business Administration and Management of the UPV in the different courses that are the control point for the assessment of the skill. The biggest challenge for lecturers is to adapt the methodology of skill assessment to the content of the course in relation to the business world and the level of domain established.
\end{abstract}

Keywords: skills, training, methodology, assessment, business, challenge.

\section{Resumen}

La UPV ha definido un perfil competencial para todos los alumnos egresados de la UPV con el fin de mejorar su desempeño profesional. Este perfil consta de 13 competencias transversales. El objetivo de este trabajo consiste en presentar distintas metodologías de evaluación de la CT-04. "Innovación, creatividad y emprendimiento", llevadas a cabo a lo largo del Grado en Administración y Dirección de Empresas (ADE) de la UPV en las distitnas asignaturas que son punto de control para la evaluación de la competencia. El mayor reto para los profesores es adaptar la metodología de evaluación de la competencia a los contenidos de la materia impartida en relación con el mundo de la empresa y al nivel de dominio establecido.

Palabras clave: competencias, formación, metodología, evaluación, empresa, reto. 


\section{Introducción}

La Universitat Politècnica de València está desarrollando el proyecto de competencias transversales UPV (Universitat Politècnica de València, 2016) que tiene como objetivo principal acreditar las competencias transversales UPV a los estudiantes egresados en cualquiera de los títulos oficiales impartidos en la Universitat Politècnica de València. Las competencias transversales UPV, que pretenden sintetizar un perfil competencial para todos los alumnos egresados de la UPV, garantizando que se cubre el marco de referencia de todas las titulaciones, son las siguientes: CT-01. Comprensión e integración, CT-02. Aplicación y pensamiento práctico, CT-03. Análisis y resolución de problemas, CT-04. Innovación, creatividad y emprendimiento, CT-05. Diseño y proyecto, CT-06. Trabajo en equipo y liderazgo, CT-07. Responsabilidad ética, medioambiental y profesional, CT-08. Comunicación efectiva, CT-09. Pensamiento crítico, CT-10. Conocimiento de problemas contemporáneos, CT-11. Aprendizaje permanente, CT-12. Planificación y gestión del tiempo, CT-13. Instrumental específica.

El marco general de evaluación-acreditación de las competencias es el siguiente:

- Vía 1. Evidencias recogidas a través de las materias/asignaturas consideradas punto de control;

- Vía 2. Cuestionarios ya definidos que se integran en la aplicación de TFG/TFM;

- Vía 3. La UPV establece el tipo de evidencias relacionadas con las CT que pueden ser reconocidas institucionalmente. Se establecerá un mecanismo de jurisprudencia donde se irán analizando y sumando todas las posibles iniciativas.

Cada competencia transversal se evalúa atendiendo a los siguientes criterios: A (la competencia se supera excelentemente), B (la competencia se alcanza completamente), C (la competencia se alcanza parcialmente), D (la competencia no se alcanza, es decir, el criterio no logra el nivel mínimo) o en blanco (no se tienen suficientes evidencias para valorarla).

Dada la situación actual, el reto al que nos enfrentamos es cómo plantear estrategias de evaluación en las materias/asignaturas consideradas punto de control que se centren en la realización, por parte de los estudiantes, de actividades lo más auténticas posibles y con un nivel adecuado de complejidad según el momento formativo y el contexto en el que se desarrolla la docencia. Fernández (2006) destaca que los métodos de enseñanza en los que participa el alumno, donde la responsabilidad del aprendizaje depende directamente de lo que él hace, por un lado, generan aprendizajes más profundos, significativos y duraderos $\mathrm{y}$, por otro lado, facilitan la transferencia a contextos más heterogéneos. De hecho, un alumno sin demasiado interés puede mejorar su rendimiento y calidad del aprendizaje cuando el docente utiliza metodologías centradas en los alumnos (Biggs, 2004).

Como la evaluación de cada competencia es diferente, en este estudio, nos centramos en la competencia 4: CT-04. "Innovación, creatividad y emprendimiento". La UPV (2018) define la innovación como la capacidad de responder de manera satisfactoria a las 
necesidades personales, organizativas y sociales, modificando procesos y/o resultados para generar nuevo valor. Al mismo tiempo, el desarrollo de esta competencia requiere, tanto el pensar de modo diferente para aportar distintas perspectivas (creatividad), como el comprometer determinados recursos por iniciativa propia, con el fin de explorar una oportunidad, asumiendo el riesgo que esto conlleva (emprendimiento).

En definitiva, el objetivo de este trabajo consiste en presentar distintas metodologías de evaluación de la CT-04. "Innovación, creatividad y emprendimiento", llevadas a cabo a lo largo del Grado en Administración y Dirección de Empresas (ADE) de la UPV en las distitnas asignaturas que son punto de control para la evaluación de la competencia.

\section{El Grado en ADE de la UPV}

El objetivo del Grado en ADE es formar profesionales capaces de gestionar, dirigir, asesorar y evaluar las organizaciones empresariales. Los graduados tendrán pues una completa formación en la gestión y organización de las empresas, lo que les permitirá desarrollar tareas de responsabilidad en el ámbito global de las organizaciones o en puestos directivos o intermedios de los diferentes departamentos que forman la empresa (producción, recursos humanos, financiación, comercialización, inversión, administración o contabilidad). Asimismo, también podrán desarrollar el libre ejercicio de la profesión como auditor/a o consultor/a, e incluso emprendiendo una nueva empresa.

Los créditos establecidos para obtener el Grado en ADE son 240 ECTS. Esos créditos se reparten en asignaturas que se realizan durante 4 cursos. Las asignaturas se clasifican en formación básica, obligatorias y optativas. Cabe la posibilidad de realizar una intensificación específica si se escogen unas optativas concretas en cuarto curso. Los alumnos, si así lo desean, pueden escoger entre "Asesoría y Servicios Financieros" u "Organización de Empresas Industriales y de Servicios".

Las asignaturas seleccionadas como punto de control para la evaluación de la competencia CT-04. "Innovación, creatividad y emprendimiento" son Derecho de la empresa, Estrategia y diseño de la organización, Contabilidad de costes e introducción a la auditoría, Diseño de servicios, de la idea a la implementación, Ecosistemas de innovación y competitividad, Emprendedores y creación de empresas y Juego de empresas (Business game).

La Tabla 1 recoge la información básica de cada una de estas asignaturas. 
Tabla 1. Asignaturas punto de control

\begin{tabular}{|c|c|c|c|}
\hline Asignatura & Curso & Créditos & Tipo \\
\hline Derecho de la empresa & Primero & 6 & $\begin{array}{c}\text { Formación } \\
\text { básica }\end{array}$ \\
\hline $\begin{array}{l}\text { Estrategia y diseño de la } \\
\text { organización }\end{array}$ & Segundo & 6 & Obligatoria \\
\hline $\begin{array}{l}\text { Contabilidad de costes e } \\
\text { introducción a la } \\
\text { auditoria }\end{array}$ & Tercero & 6 & Obligatoria \\
\hline $\begin{array}{c}\text { Diseño de servicios, de } \\
\text { la idea a la } \\
\text { implementación }\end{array}$ & Cuarto & 4,5 & Optativa \\
\hline $\begin{array}{l}\text { Ecosistemas de } \\
\text { innovación y } \\
\text { competitividad }\end{array}$ & Cuarto & 4,5 & Optativa \\
\hline $\begin{array}{c}\text { Emprendedores y } \\
\text { creación de empresas }\end{array}$ & Cuarto & 4,5 & Optativa \\
\hline $\begin{array}{c}\text { Juego de empresas } \\
\text { (Business game) }\end{array}$ & Cuarto & 4,5 & Optativa \\
\hline
\end{tabular}

Fuente: Elaboración propia.

\section{Metodologías de evaluación}

\subsection{Niveles de dominio}

Para cada competencia transversal, existen 3 niveles de dominio: Nivel 1 (asignaturas de primero y segundo de grado), nivel 2 (asignaturas de tercero y cuarto de grado) y nivel 3 (asignaturas de máster). En nuestro caso, sólo vamos a explicar los niveles 1 y 2 ya que nos centramos en el Grado en ADE.

En el nivel 1, el objetivo es que el alumno se cuestione la realidad, identificando necesidades de mejora e ideas que puedan generar valor. Los indicadores a utilizar son: cuestionarse la realidad, aportar ideas, plasmar de manera formal las ideas e identificar los resultados.

En el nivel 2, el objetivo es que el alumno sea capaz de aportar ideas y planteamientos originales que aporten valor, a través de estrategias y técnicas de creatividad. Los indicadores propuestos son: identificar oportunidades y/o aspectos de mejora, aportar ideas y planteamientos originales, emplear estrategias y/o técnicas creativas para plasmar de manera formal las ideas y soluciones y controlar resultados. 
La UPV (2018) propone las siguientes actividades formativas para el desarrollo de esta competencia: actividades de emprendimiento, análisis DAFO, juego y simulación, problemas, proyectos y técnicas de creatividad (generación de ideas, pensamiento visual...). Para evaluar esta competencia, se pueden utilizar procedimientos y técnicas de evaluación como: observación, autoevaluación, evaluación entre iguales, exposición oral, portafolio y redacción de informes escritos, individuales o en grupo. También es recomendable el uso de rúbricas para objetivar los procesos evaluativos.

A continuación, se muestra la metodología de evaluación de cada una de las asignaturas que son punto de control de la CT-04. "Innovación, creatividad y emprendimiento" en el Grado en ADE de la UPV.

\subsection{Derecho de la empresa}

Esta asignatura abarca un amplio abanico de temas legales relacionados con la empresa: estatuto jurídico del empresario mercantil, derecho de sociedades, las sociedades personalistas, las sociedades capitalistas, el derecho de la competencia, la propiedad privada, los contratos, los instrumentos de pago y la insolvencia patrimonial. La asignatura no exige conocimientos previos de otras asignaturas ya que se imparte en el primer curso del Grado en ADE.

La competencia CT-04. "Innovación, creatividad y emprendimiento" se evalúa a partir de las prácticas de laboratorio sobre los pasos y documentos jurídicos necesarios para crear una empresa y proteger los resultados de la actividad de innovación. Concretamente, los alumnos aprenden los pasos prácticos (reserva de denominación social, que no haya una marca anterior, búsqueda de escritura y estatutos tipo, CNEA) para constituir una SA y una SL., así como a buscar en las webs de la OEPM, EUIPO y EPO las marcas, diseños y modelos industriales, patentes y modelos de utilidad, para proteger sus creaciones industriales e intelectuales y, sobre todo, trabajar con innovaciones que no estén ya protegidas por otros. En concreto, en 2GIA aprenden a ver las ventajas entre la protección del software por derechos de autor y como patente, para ello se han manejado principalmente con la base de datos SPACENET. Como criterio de evaluación, el profesor comprueba que el alumno realiza la práctica y resuelve el caso de manera adecuada.

\subsection{Estrategia y diseño de la organización}

La asignatura pretende que el alumno adquiera el conocimiento de los fundamentos conceptuales básicos de la dirección estratégica de la empresa, familiarizándose con los conceptos y terminología específica de la disciplina: qué es la estrategia y la dirección estratégica y el importante papel que la dirección estratégica desempeña dentro de la empresa. Además, se profundiza en las características básicas de distintas estrategias competitivas que pueden adoptar las empresas para comprender con claridad los problemas y planteamientos estratégicos a los que se enfrentan en situaciones reales las empresas. En todo este proceso, se busca que el alumno comprenda la interacción que se produce entre la estrategia de la empresa y los elementos sociales y estructurales de la 
misma para así conocer las circunstancias que permiten o impiden transformar las estrategias elegidas en acciones de la empresa.

La competencia CT-04. "Innovación, creatividad y emprendimiento" se evalúa mediante las siguientes actividades:

- Estudio de casos relacionados con emprendedores, innovación y creatividad: Caso Dyson, Caso Virgin, Caso Ebay.

- Realización de trabajos en grupo relacionados con emprendedores, innovación y creatividad.

- Trabajo sobre la estrategia de una empresa (Zara) donde los alumnos deben de desarrollar el modelo de negocio de una empresa de éxito basándose en los ejemplos proporcionados por el profesor.

La evaluación de esta competencia se realiza a través de la evaluación de los casos realizados en el aula y del trabajo presentado por los alumnos midiendo el grado de creatividad de las soluciones propuestas a las preguntas planteadas, grado en el que el alumno es capaz de aportar ideas originales al problema planteado en el caso, grado en el que el alumno es capaz identificar las oportunidades y aspectos de mejora y de proponer planes de acción para dar solución a los problemas planteados en el caso. Como conclusion, los casos y trabajos planteados permiten valorar la importancia de la innovación, identificar oportunidades y aspectos de mejora, proponer planes de acción o aportar ideas originales.

\subsection{Contabilidad de costes e introducción a la auditoria}

La asignatura "Contabilidad de costes e introducción a la auditoria" presenta al alumno la delimitación de la contabilidad de gestión y de costes, el comportamiento y clasificación de los costes, cómo tomar decisiones a partir de los costes calculados, el concepto de auditoría, los distintos tipos de auditoría, normas y planificación, qué es la auditoría de Cuentas Anuales y el informe de auditoría de cuentas anuales.

La competencia CT-04. "Innovación, creatividad y emprendimiento" se evalúa mediante una práctica de laboratorio sobre los costes de crear una empresa y la importancia de alcanzar el punto de equilibrio. Es un ejercicio numérico que pone claramente de manifiesto la importancia de controlar los costes en los planes de empresa.

\subsection{Diseño de servicios, de la idea a la implementación}

Este asignatura cubre el diseño de propuestas de valor en servicios a través de un enfoque centrado en el cliente. Usando diferentes herramientas teóricas, los estudiantes aprenden cómo analizar, mejorar y diseñar servicios para empresas en diferentes industrias.

La evaluación de la competencia CT-04. "Innovación, creatividad y emprendimiento" se realiza mediante el uso de diferentes herramientas relacionadas con la innovación y el emprendimiento a través de ejercicios y casos. Cada tarea incluye la aplicación de una o más herramientas relacionadas con la innovación y el espíritu empresarial. En total, son 
8 tareas: 2 individuales y el resto en grupo. La competencia se evalúa a partir de la nota final calculada de una media ponderada de las tareas.

\subsection{Ecosistemas de innovación y competitividad}

El objetivo de la asignatura consiste en conocer y analizar las estrategias y políticas de innovación y competitividad en distintos niveles, i.e. país, región, clusters y empresas así como su impacto en el crecimiento y la competitividad de los mismos. Se analizan las políticas industriales y de innovación y los mecanismos para su diseño, implementación y evaluación. Además, se introduce el concepto de competitividad y se analiza como factor estructural de un país y de una región, analizando con detalle la competitividad generada en clústeres industriales, distritos industriales y otros ecosistemas de innovación (Universidades, aceleradoras, etc.).

La evaluación de la competencia CT-04. "Innovación, creatividad y emprendimiento" se realiza mediante el conocimiento del alumno de los problemas actuales de la innovación. Para ello, el alumno elabora un informe sobre un hecho contemporáneo y actual relacionado con la innovación y aporta una reflexión y descripción del mismo. El profesor evalúa la competencia a partir de la exposición oral del informe y el informe en sí mismo.

\subsection{Emprendedores y creación de empresas}

La asignatura trata de promover el espíritu empresarial entre los alumnos. Para ello, se les orienta en el uso de herramientas, a seleccionar la forma jurídica de la empresa, a obtener financiación, a realizar un plan de empresa y a analizar la experiencia de otros emprendedores. Igualmente, se presenta a los alumnos las aceleradoras e incubadoras de negocios, las empresas de base tecnológica y spin off, las start up y viveros de empresa y los simuladores de modelos de negocio.

La competencia CT-04. "Innovación, creatividad y emprendimiento" se evalúa a partir del Plan de Empresa realizado y presentado por los alumnos. En el sistema de evaluación, supone el $40 \%$ de la nota final y el 100\% para la evaluación de la competencia.

\subsection{Juego de empresas (Business game)}

Esta asignatura es totalmente práctica, siguiendo la metodología basada en el "learning by doing" (aprender haciendo), siempre a través de grupos de trabajo, con plazos limitados, simulando las condiciones de trabajo en las organizaciones en donde trabajarán posteriormente los alumnos. Los objetivos de la asignatura son: Dotar al alumnado de los conocimientos y habilidades necesarios para tomar decisiones estratégicas en equipo, acercar al alumnado a situaciones cuasi-reales de toma de decisión empresarial, potenciar en los alumnos el uso de nuevas tecnologías de información, como son la utilización de un simulador de juego de empresa y el manejo del correo electrónico.

La evaluación de la competencia CT-04. "Innovación, creatividad y emprendimiento" se realiza mediante la participación en un Business Game. Los alumnos constituyen empresas que participan en un simulador que escenifica el funcionamiento real de una empresa en interacción con sus competidores, mercado y entorno. Como criterio de 
evaluación, el alumno realiza informes escritos, así como, presenta y hace una puesta en común de las áreas funcionales de la empresa.

\section{Conclusiones}

La UPV está apostando por la formación de sus alumnos en competencias transversales para que puedan desenvolverse mejor en el mundo laboral. Para su evaluaciónacreditación, se designan distintas asignaturas que son punto de control de cada una de las 13 competencias transversales definidas por la UPV. Esta apuesta supone un reto para los profesores ya que hemos de adaptar nuestras estrategias de evaluación a las distintas competencias transversales.

Con la competencia CT-04. "Innovación, creatividad y emprendimiento", entendemos que innovar es responder, de manera satisfactoria y original, a las necesidades y demandas personales, organizativas y sociales con una actitud emprendedora. La UPV establece los distintos niveles de dominio y propone diferentes metodologías de evaluación.

En el Grado en ADE, los profesores responsables de las asignaturas que son punto de control de la competencia CT-04. "Innovación, creatividad y emprendimiento" utilizan diferentes metodologías de evaluación. Su objetivo principal es adaptar la metodología de evaluación de la competencia a los contenidos de la materia impartida en relación con el mundo de la empresa y al nivel de dominio establecido. Todos los profesores de estas asignaturas utilizan metodologías de evaluación propuestas por la UPV para la evaluación de dicha competencia, teniendo en cuenta los objetivos a conseguir en cada nivel.

Dada la relación de las asignaturas con el mundo de la empresa, las mismas asignaturas se adaptan fácilmente a la evaluación de esta competencia. Esto supone una gran ventaja del Grado en ADE frente a otros posibles grados en la UPV. Además, la evaluación de la competencia se ajusta a los criterios para cada uno de los niveles de dominio propuestos por la UPV. En este sentido, cabe destacar que es clave que haya una coordinación entre los profesores de los niveles 1 y 2 para poder así constatar la evolución del alumno a lo largo del Grado en ADE.

Como inconvenientes, algunos profesores responsables de las asignaturas destacan la falta de tiempo para poder tratar el tema con mayor profundidad. Por ejemplo, algunos sugieren poder disponer de más tiempo para la exposición oral de los trabajos, o bien, para la realización del trabajo autónomo. En el caso de evaluar tareas concretas, el inconveniente de elegir unas cuantas es si el alumno no realiza todas ellas. Ante este problema, se podría animar al alumno a realizar las elegidas para la evaluación de la competencia, o bien, contar con una tarea comodín o extra para poder evaluarla. 


\section{Referencias}

BIGGS, J. (2004). Calidad del aprendizaje universitario. Madrid: Narcea Ediciones.

FERNÁNDEZ, A. (2006). "Metodologías activas para la formación de competencias” en Educatio siglo XXI, vol. 24, p. 35-56.

UNIVERSITAT POLITÈCNICA DE VALÉNCIA (2016). Competencias transversales. $<$ http://www.upv.es/contenidos/COMPTRAN/indexc.html $>$ [Consulta: 25 de mayo de 2019].

UNIVERSITAT POLITÈCNICA DE VALÉNCIA (2018). Innovación, creatividad y emprendimiento.

$<$ http://www.upv.es/contenidos/COMPTRAN/info/954818normalc.html $>$ [Consulta: 25 de mayo de 2019]. 